\title{
A Firenze, c'è chi si arregge
}

\author{
Neri Binazzi
}

PUBBLICATO: 29 MARZO 2019

\section{Quesito:}

Alcuni lettori soprattutto fiorentini ci chiedono se arreggere e arreggersi siano toscanismi o forme italiane. Un lettore di Roma ci chiede se il loro impiego in luogo di reggere e reggersi sia da considerarsi scorretto.

\section{A Firenze, c'è chi si arregge}

«C’è scritto anche sugli autobus: "arreggersi agli appositi sostegni"». L'affermazione, registrata in occasione di un lavoro orientato a misurare la percezione del grado di "esportabilità extratoscana" di una selezione del lessico d'uso quotidiano, appare profondamente rivelatrice di una considerazione della voce che ancora oggi è assai poco investita, a Firenze, di sospetti riguardo alla propria cittadinanza, che invece è tipicamente locale. A questa debole marcatezza in senso "dialettale" corrisponde un particolare radicamento della voce: tra i giovani studiati da Binazzi 1997 arreggersi è dichiarato normalmente in uso da circa l'8o\% del campione.

Nel sostenere la percezione della voce come sostanzialmente "italiana" ha evidentemente un peso rilevante il fatto che la forma si configuri come una variante formale di reggere (evidentemente, e giustamente, sentita al riparo di ogni "sospetto di dialettalità"), con cui oltretutto arreggere condivide l'intero spettro semantico, anche nel gettonatissimo riflessivo, con il valore di 'sostenersi, afferrarsi per non cadere' (a cui fa riferimento anche il lettore che, tra quelli che ci hanno ha chiesto informazioni sulla forma, ha definito arreggersi 'tenersi aggrappato a qualcosa'), e che costituisce il riferimento dell'affermazione iniziale da cui abbiamo preso le mosse. Un riferimento che è anche quello restituito dalla lessicografia vernacolare: Rosi Galli 2009, per esempio, dopo aver proposto, per arreggere, il significato 'reggere, tenere', propone come esempio l'espressione esortativa Arreggiti bene 'tieniti ben stretto'; su questa linea si colloca anche Capanni 2oII, che riconnette al significato 'reggere, tenere, appigliarsi' l'esempio d'uso La s'arregga per benino 'Si tenga ben stretto'.

In realtà l'avvertenza ai passeggeri che il parlante ricordato all'inizio aveva "riletto" come arreggersi agli appositi sostegni presentava, in realtà, sorreggersi, che è la scelta prevista ancora oggi dal testo delle condizioni di trasporto dell'azienda che a Firenze si occupa della mobilità in ambito metropolitano:

In generale, per la propria ed altrui incolumità, è vietato [...] viaggiare senza sorreggersi agli appositi sostegni durante la marcia delle vetture (dalla pagina"Condizioni di trasporto" sul sito ataf.net).

Per fare qualche esempio in giro per l'Italia, sorreggersi è anche la scelta "aziendale" di Napoli, che tra le norme di comportamento che i viaggiatori devono rispettare su bus e tram (art. 2), avverte che

è obbligatorio sorreggersi agli appositi sostegni durante la marcia della vettura (dalla pagina "Regolamento bus e tram" sul sito anm.it).

Per i viaggiatori della metropolitana milanese, invece, l'azienda sceglie un approccio meno perentorio e più discorsivo, mirato al coinvolgimento consapevole di un pubblico che non è unentità astratta ma qualcuno a cui ci si rivolge direttamente, quasi confidenzialmente. In questo contesto l'indicazione del comportamento più idoneo non si orienta su una sola scelta, ma prevede una variatio (prima tenersi, poi, per introdurre la formula rituale, reggersi):

Sul treno, se stai in piedi, tieniti sempre ben saldo alle piantane di sostegno o ai corrimano. Assicurati che anche i bambini che sono insieme a te lo facciano. Non reggersi agli appositi sostegni è rischioso: la disattenzione può infatti causare cadute (dalla pagina "Viaggiare in sicurezza" sul sito atm.it).

A proposito di ritualità, da formula "aziendalese" reggersi agli appositi sostegni sembra divenuta ormai una frase fatta di pubblico dominio, a cui si chiede di esprimere - anche in prospettiva figurata - un'azione la cui 
familiarità è, intrinsecamente, quella con la formula che la esprime. Reggersi agli appositi sostegnil, così, è il nome che la rivista "Quattroruote" ha scelto per un forum di discussione tra automobilisti. Addirittura, nel giugno 2008 il sito web "Antologia Politica" propose un articolo in cui la nostra formula veniva evocata per commentare il percorso (ritenuto, evidentemente, traballante) che avrebbe dovuto condurre alla creazione di un nuovo soggetto politico:

\section{SI PREGA DI REGGERSI AGLI APPOSITI SOSTEGNI}

\section{IL VIAGGIO VERSO IL "POPOLO DELLE LIBERTA" SI ANNUNCIA MENO CONFORTEVOLE DEL PREVISTO (dal blog antologiapolitica.wordpress.com).}

Ma torniamo ora a Firenze, dove è comunissimo arreggersi per non perdere l'equilibrio, ma dove si arregge anche qualcosa o qualcuno che, precariamente in equilibrio, potrebbe finire in terra ("mi arreggi un momento la bicicletta?"; "guarda, arrèggilo, perché casca in terra dal sonno"). Oppure si arregge qualcosa che non si vuole appoggiare ("dammi, te l'arreggo io la borsa mentre cerchi le chiavi").

Lo spettro semantico del verbo, come si vede, è sostanzialmente sovrapponibile con quello coperto da reggere / reggersi e tenere/ tenersi. Questi, a loro volta, possono avvalersi del supporto di un prefisso so- (che ha per antenato il latino sub-) che, a partire dall'originaria indicazione dello svolgersi dal basso dell'operazione (la trave sorregge / sostiene il tetto), di per sé rintracciabile anche negli usi figurati, peraltro finalizzati a esprimere il concetto di 'essere d'aiuto' (lo sorregge una volontà di ferro; bisogna sostenere l'economia), tende progressivamente a produrre forme sostanzialmente sinonimiche, in cui si può magari rilevare una sfumatura intensiva (se non lo sorreggi / sostieni, puó cadere). Di fatto, anche arreggere deve la sua configurazione formale all'intervento di un prefisso (che in quanto tale costituisce, in diacronia, l'esito della preposizione latina $a d-$ ), che, in genere adottato per formare verbi parasintetici a partire da sostantivi e aggettivi (latte > allattare; bello > abbellire), può anche unirsi a forme verbali, producendo in questo caso varianti che rispetto alla forma originaria possono presentare una diversa sfumatura semantica (rischiare $>$ arrischiarsi).

Da questo punto di vista la particolarità della forma fiorentina riguarda il fatto che, rispetto alla forma "non prefissata", arreggere non solo non presenta deviazioni semantiche, ma non pare sostanzialmente disponibile a usi figurati, che sono esclusi, si direbbe, proprio la presenza del prefisso (non è dunque possibile, a Firenze, "non lo arreggo più'" "non lo reggo / sopporto più').

Questa particolare "concretezza semantica" della voce trova forse la sua chiave di lettura più convincente se inquadriamo arreggere / arreggersi nel panorama delle voci che, tradizionali nell'uso di Firenze, si presentano di fatto come forme esito di prefissazione. Nella banca dati del Vocabolario del fiorentino contemporaneo in corso di pubblicazione presso l'Accademia della Crusca, dove naturalmente arreggere è previsto, troviamo per esempio abbadare 'porre attenzione', usato soprattutto in formulazioni esortative, anche transitivamente ("oh, abbadaci! Facci attenzione!"; "abbadami la pentola, vo un attimo giù"; "abbadami il bambino: non lo lasciare solo"); oppure ammollare 'smettere di discutere, desistere da un proposito' ("l'è meglio ammollare, sennò sai quanto la si farebbe lunga!»; «Facciamola finita, facciamo festa: ammolliàmola”). In quanto tali, le forme in questione si configurano come prefissate rispetto alle corrispondenti badare e mollare, le quali, riguardo ai valori indicati ('prestare attenzione'; 'rinunciare a un proposito') hanno cittadinanza, magari con sfumature in parte diverse, anche nell'italiano comune (che invece non prevede abbadare e ammollare).

Del resto, considerando ora varianti allo stesso modo "italiane", l'uso fiorentino tradizionale preferisce arrovesciare a rovesciare, assomigliare a somigliare.

A proposito di arrovesciare, andrà osservato che la modalità prefissata ricorre anche nella locuzione avverbiale all'arrovéscia 'al contrario' (usata sia in senso proprio: il berretto messo all'arrovescia; sia in senso figurato: lui fa tutto all'arrovescia). Analogamente, il fiorentino preferisce addirizzare a 'raddrizzare', sebbene in questo caso, come succede per arreggersi, la modalità più gettonata a Firenze non pare disponibile a usi figurati (quindi si addirizza un chiodo ma non, per esempio, un torto subito). Uscendo un momento dal sistema verbale, una sorta di predisposizione fiorentina all'estensione di forme per il contributo di $a$ - la possiamo individuare anche, ascoltando il parlato dei più anziani, in aggettivi come abbasito, perfettamente confrontabile con l'italiano basito 'impietrito (negativamente) dallo stupore', o come affortunato 'fortunato'. 
Quanto alla questione dell'italianità di arreggere / arreggersi, essa è esclusa: i vocabolari dell'italiano in pratica non lo prevedono (mentre lo troviamo, anche se non sistematicamente, nei lessici del fiorentino: cfr. anche Camaiti 1934), e sebbene i parlanti fiorentini possano ritenersi sicuri che, invitando un non toscano ad arreggersi per non cadere, quello capirà, essi devono anche sapere che, pur apprezzando la premura, quel non toscano difficilmente condividerà allo stesso modo la scelta diarreggere, in cui invece troverà un'ulteriore conferma dell'abitudine dei fiorentini a prendere poco in considerazione l'eventualità che il proprio parlato quotidiano possa non essere "automaticamente" italiano.

Lo stesso verbo, con la $r$ scempia propria di tutti prefissati del genere, è diffusissimo anche a Roma, dove ha le stesse connotazioni in senso dialettale: il vocabolario di Chiappini (1933) registra "Arrégge, Arégge, Reggere, detto anche del tempo" (che si mantiene sereno); il dizionario di Ravaro (1994) solo arègge, con molte collocazioni e polirematiche che ne ampliano la gamma semantica. Areggi questo, nel senso di 'tieni questa cosa in mano tu', mentre io faccio un'altra cosa, è un imperativo ben diffuso, così come espressioni del tipo quella chi la regge? 'nessuno la sopporta'!

\section{Nota bibliografica:}

- Neri Binazzi, Le parole dei giovani fiorentini: variazione linguistica e variazione sociale, Roma, Bulzoni, 1997.

- Venturino Camaiti, Dizionario etimologico, pratico-dimostrativo, del linguaggio fiorentino, Firenze, Vallecchi, 1934.

- Roberta Capanni (a cura di), Il vero Vohabolario del vernaholo fiorentino, Firenze, Romano Editore, zorI

- Filippo Chiappini, Vocabolario romanesco, Roma, Leonardo Da Vinci, I933.

- Teresa Poggi Salani, Neri Binazzi, Matilde Paoli, Cristina Torchia (a cura di), Parole di Firenze dal Vocabolario del fiorentino contemporaneo, Firenze, Accademia della Crusca 2012.

- Fernando Ravaro, Dizionario romanesco, Roma, Newton Compton, 1994.

- Stefano Rosi Galli, Vohabolario del Vernaholo Fiorentino e del Dialetto Toscano di ieri e di oggi, Firenze, Romano Editore, 2009.

\section{Cita come:}

Neri Binazzi, A Firenze, c'è chisi arregge , "Italiano digitale", 2019, VIII, 2019/1 (gennaio-marzo) , pp. 53-55.

DOI: $10.35948 / 2532-9006 / 2019.3081$

\section{Copyright 2019 Accademia della Crusca}

Pubblicato con licenza creative commons CC BY-NC-ND 